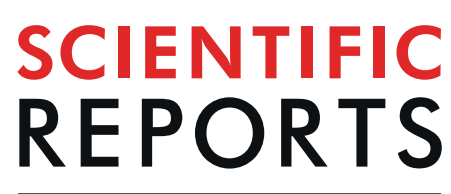

natureresearch

\title{
OPEN Evaluation of (-)-epicatechin metabolites as recovery biomarker of dietary flavan-3-ol intake
}

Received: 29 July 2019

Accepted: 29 August 2019

Published online: 11 September 2019
Javier I. Ottaviani ${ }^{1}$, Reedmond Fong ${ }^{2}$, Jennifer Kimball ${ }^{2}$, Jodi L. Ensunsa ${ }^{2}$, Nicola Gray ${ }^{3}$, Anna Vogiatzoglou ${ }^{4}$, Abigail Britten ${ }^{5}$, Debora Lucarelli ${ }^{5}$, Robert Luben ${ }^{6}{ }^{6}$, Philip B. Grace ${ }^{7}$, Deborah H. Mawson ${ }^{7}$, Amy Tym ${ }^{7}$, Antonia Wierzbicki ${ }^{7}$, A. David Smith ${ }^{8}$, Nicholas J. Wareham ${ }^{5}$, Nita G. Forouhi ${ }^{5}{ }^{5}$, Kay-Tee Khaw ${ }^{6}$, Hagen Schroeter ${ }^{1}$ \& Gunter G. C. Kuhnle $\mathbb{1 i}^{3,6}$

Data from dietary intervention studies suggest that intake of (-)-epicatechin mediates beneficial vascular effects in humans. However, population-based investigations are required to evaluate associations between habitual intake and health and these studies rely on accurate estimates of intake, which nutritional biomarkers can provide. Here, we evaluate a series of structurally related (-)-epicatechin metabolites (SREM), particularly (-)-epicatechin-3'-glucuronide, (-)-epicatechin3'-sulfate and 3'-O-methyl-(-)-epicatechin-5-sulfate $\left(\mathrm{SREM}_{\mathrm{B}}\right)$, as flavan-3-ol and (-)-epicatechin intake. SREM $M_{B}$ in urine proved to be a specific indicator of $(-)$-epicatechin intake, showing also a strong correlation with the amount of $(-)$-epicatechin ingested $\left(R^{2}: 0.86(95 \% \mathrm{Cl} 0.81 ; 0.92)\right.$. The median recovery of (-)-epicatechin as SREM in $^{2} 4 \mathrm{~h}$ urine was $10 \%$ (IQR 7-13\%) and we found $\mathrm{SREM}_{B}$ in the majority of participants of EPIC Norfolk (83\% of 24,341$)$ with a mean concentration of $2.4 \pm 3.2$ $\mu \mathrm{mol} / \mathrm{L}$. Our results show that $\mathrm{SREM}_{B}$ are suitable as biomarker of (-)-epicatechin intake. According to evaluation criteria from IARC and the Institute of Medicine, the results obtained support use of SREM ${ }_{B}$ as a recovery biomarker to estimate actual intake of (-)-epicatechin.

There is considerable evidence suggesting that flavanols can be beneficial for cardiovascular disease prevention and health maintenance, although current data are still insufficient to provide general dietary guidance ${ }^{1,2}$. Recent data have shown that the monomer (-)-epicatechin, but not oligomeric and polymeric procyanidins, are responsible for improvements in vascular function ${ }^{3}$. While the effect of $(-)$-epicatechin on health can be investigated in clinical dietary intervention studies, only epidemiological investigations can provide population-based information about the long-term effect of (-)-epicatechin as part of the habitual diet. However, such investigations rely on an accurate estimate of intake, and this is not possible with the methods commonly used today. Reasons lie in the significant limitation of self-reported dietary data as well as in the large variability of the (-)-epicatechin content of foods (Table 1). Alternatives to the latter approach emerge in the use of nutritional biomarkers of intake, which reflect actual intake and do not rely on self-reported food intakes and food composition data ${ }^{4,5}$. While we have recently evaluated a nutritional biomarker of flavan-3-ol intake ${ }^{6}$, there are currently no established biomarkers to specifically assess the intake of (-)-epicatechin.

Nutritional biomarkers for phenolic compounds such as (-)-epicatechin, are often based on the concentration of their metabolites in blood or urine $e^{7}$. However, a careful evaluation of phenolic metabolites is important to assess their suitability and to identify their strengths and limitations as biomarkers of intake. Previously, we used the evaluation process proposed by IARC (International Agency for Research on Cancer) and the IOM (Institute of Medicine $)^{6}$ to evaluate 5- $\left(3^{\prime}, 4^{\prime}\right.$-dihydroxyphenyl)- $\gamma$-valerolactone (gVL) and its metabolites (gVLM) as nutritional biomarker of flavan-3-ol intake $e^{6}$. These criteria include: (i) demonstration of accuracy, precision

${ }^{1}$ Mars, Inc., McLean, VA, USA. ${ }^{2}$ Department of Nutrition, UC Davis, Davis, CA, USA. ${ }^{3}$ Department of Food and Nutritional Sciences, University of Reading, Reading, UK. ${ }^{4}$ School of Life Sciences, Coventry University, Coventry, UK. ${ }^{5}$ MRC Epidemiology Unit, University of Cambridge, Cambridge, UK. ${ }^{6}$ Department of Public Health and Primary Care, University of Cambridge, Cambridge, UK. ${ }^{7}$ LGC, Newmarket Road, Fordham, UK. ${ }^{8}$ OPTIMA Department of Pharmacology, University of Oxford, Oxford, UK. Correspondence and requests for materials should be addressed to G.G.C.K. (email: g.g.kuhnle@reading.ac.uk) 


\begin{tabular}{|l|l|}
\hline Food & Content [mean \pm SD, range] \\
\hline Apple (Cider), peeled & $28.7 \pm 26.5(0.0-141.0)$ \\
\hline Apple (Dessert), peeled & $6.7 \pm 4.5(0.0-19.8)$ \\
\hline Apricot, raw & $3.5 \pm 4.3(0.0-6.1)$ \\
\hline Blackberry, raw & $11.5 \pm 10.9(2.7-18.1)$ \\
\hline Chocolate, dark & $70.4 \pm 29.5(32.7-125.0)$ \\
\hline European cranberry & $4.2 \pm 0.0(4.2-4.2)$ \\
\hline Grape (Black) & $5.2 \pm 5.6(0.7-8.6)$ \\
\hline Nectarine, peeled & $3.0 \pm 1.1(1.3-5.6)$ \\
\hline Peach, peeled & $8.0 \pm 4.2(0.7-16.5)$ \\
\hline Pear, whole & $3.8 \pm 2.7(0.2-7.5)$ \\
\hline Red raspberry, raw & $5.0 \pm 3.8(0.3-8.3)$ \\
\hline Sweet cherry, raw & $7.8 \pm 2.9(5.5-9.5)$ \\
\hline Tea (Black), infusion & $3.9 \pm 4.3(0.0-16.8)$ \\
\hline Tea (Green), infusion & $7.9 \pm 13.7(0.0-73.9)$ \\
\hline Tea (Oolong), infusion & $2.7 \pm 3.8(0.0-13.2)$ \\
\hline Wine (Red) & $3.8 \pm 3.2(0.0-16.5)$ \\
\hline
\end{tabular}

Table 1. Ranges of $(-)$-epicatechin content $(\mathrm{mg} / 100 \mathrm{~g}$ or $\mathrm{mg} / 100 \mathrm{~mL})$ of different foods commonly consumed (data from Phenol Explorer 3.0 $0^{37}$ ).

and reliability of the analytical method to quantify the candidate biomarker; (ii) demonstration and characterization of the relationship between intake and candidate biomarker levels in urine, including characterizing specificity and intra-individual variability; and (iii) demonstration of the applicability of the candidate biomarker to estimate intake in large cohort studies. Here, we apply the same criteria to evaluate three of the most abundant structurally related (-)-epicatechin metabolites (SREM), including (-)-epicatechin-3'-glucuronide $\left(\mathrm{E} 3^{\prime} \mathrm{G}\right),(-)$-epicatechin-3'-sulfate $\left(\mathrm{E} 3^{\prime} \mathrm{S}\right)$ and $3^{\prime}-\mathrm{O}$-methyl-(-)-epicatechin-5-sulfate (3MeE5S), as biomarker of $(-)$-epicatechin intake and to estimate $(-)$-epicatechin intake in large-scale cohort studies using this biomarker.

\section{Methods}

Analytical method - epidemiological study. Analysis was performed using ultra high-performance liquid chromatography coupled to tandem mass spectrometry (UPLC-MS/MS; Waters Acquity BSM, coupled with an Applied Biosystems API 4000; see Supplemental Fig. 1 for a typical chromatogram). De novo synthesized authentic standards $s^{8,9}$ of three human structurally related epicatechin metabolites (SERMs) considered in this study $\left((-)\right.$-epicatechin-3'-glucuronide (E3'G), (-)-epicatechin-3'-sulfate (E3'S) and $3^{\prime}$-O-methyl-(-)epicatechin-5-sulfate (3Me5S)), as well as stable isotope labelled internal standards (ISTD, $\mathrm{D}_{2} / \mathrm{D}_{3}$-epicatechin$3^{\prime}$ - $\beta$-D-glucuronide, 50:50 mix) were obtained from Analyticon (Analyticon Technologies AG, Lichtenfels, Germany; Supplemental Fig. 2 for structures). Purity was assessed by LC-UV and LC-MS, and stock solutions were adjusted accordingly. Stock solutions were prepared in ethanol:water $(70: 30, \mathrm{v} / \mathrm{v})$ and stored at $-80^{\circ} \mathrm{C}$.

Flavan-3-ol metabolite-free spot urine was obtained from human volunteers on a low-flavan-3-ol diet, excluding in particular tea, pome fruits and berries from their diet. This study was approved by the University of Reading Research Ethics Committee. All participants gave their written informed consent to participate and all experiments were performed in accordance with relevant University guidelines and regulations and followed GCP (good clinical practice).

Urine samples $(60 \mu \mathrm{L})$ and internal standard solutions $(1.0 \mu \mathrm{M}$ ISTD, $60 \mu \mathrm{L})$ were combined, filtered (Impact Protein Precipitation filter plate, Phenomenex, Macclesfield, UK) by centrifugation $(500 \times \mathrm{g})$ for 2 minutes at room temperature and stored at $-20^{\circ} \mathrm{C}$ until analysis. Sample preparation was automated for the analysis of EPIC (European Prospective Investigation into Cancer) Norfolk cohort urine samples using a Hamilton Star robot (Hamilton, Bonaduz, Switzerland).

Samples were separated by liquid chromatography (Acquity, Waters, Elstree, UK) using a C18 column (Kinetex C18 $100 \times 2.1 \mathrm{~mm}, 1.7 \mu \mathrm{m}$, with $0.5 \mu \mathrm{m}$ Krudcatcher, Phenomenex, Macclesfield, UK). Gradient elution was performed at a flow rate of $0.5 \mathrm{~mL} / \mathrm{min}$ using $0.1 \%$ formic acid in water as mobile phase $\mathrm{A}$ and acetonitrile/ methanol $(9: 1, \mathrm{v} / \mathrm{v})$ as mobile phase B. The initial starting conditions were $95 \% \mathrm{~A}$, which were held for $2 \mathrm{~min}$, decreasing to $85 \% \mathrm{~A}$ at $3.5 \mathrm{~min}, 79.6 \%$ at 6.0 minutes $5 \% \mathrm{~A}$ at 6.1 minutes and held until 7.0 minutes to wash the column before returning to $95 \% \mathrm{~A}$ at 7.1 minutes for re-equilibration until 8 minutes. The column temperature was maintained at $25^{\circ} \mathrm{C}$ and the injection volume was $10 \mu \mathrm{L}$.

Compounds were detected using an Applied Biosystems Sciex API 4000 instrument (Warrington, UK) equipped with Turbo Ion Spray probe operating in negative ion mode using the parameters shown in Table 2. The spray voltage was $-4500 \mathrm{~V}$ and the source temperature was $600^{\circ} \mathrm{C}$. The dwell time was $20 \mathrm{~ms}$ for each transition.

Samples quantified using calibration standards prepared in flavan-3-ol metabolite-free urine samples (standard concentrations $[\mu \mathrm{M}]$ were prepared at $0.1,0.25,1,2,3,4,5) . \mathrm{E} 3^{\prime} \mathrm{G}, \mathrm{E} 3^{\prime} \mathrm{S}$ and $3 \mathrm{Me} 5 \mathrm{~S}$ were quantified using the peak area ratio of analyte and ISTD $\left(\mathrm{D} 2 / \mathrm{D} 3-\mathrm{E} 3^{\prime} \mathrm{G}, 50: 50(\mathrm{w} / \mathrm{w})\right.$, where the $\mathrm{D} 3-\mathrm{E} 3^{\prime} \mathrm{G}$ precursor/product ions were used). 


\begin{tabular}{|l|l|l|l|}
\hline Analyte & $\begin{array}{l}\text { Precursor ion } \\
(\mathbf{m} / \mathbf{z})\end{array}$ & $\begin{array}{l}\text { Product ion } \\
(\mathbf{m} / \mathbf{z})\end{array}$ & $\begin{array}{l}\text { Retention } \\
\text { time (min) }\end{array}$ \\
\hline (-)-epicatechin-3'-glucuronide (E3'G) & 464.8 & 289.1 & 4.2 \\
\hline (-)-epicatechin-3'-sulfate (E3'S) & 369.1 & 289.1 & 4.3 \\
\hline $3^{\prime}$-O-methyl-(-)-epicatechin-5-sulfate (3Me5S) & 382.9 & 303.1 & 4.6 \\
\hline $\mathrm{D}_{2}$-epicatechin-3'- $\beta$-D-glucuronide (D2-E3'G) & 466.6 & 291.1 & 4.1 \\
\hline $\mathrm{D}_{3}$-epicatechin-3'- $\beta$-D-glucuronide (D3-E3'G) & 468.3 & 292.1 & 4.1 \\
\hline
\end{tabular}

Table 2. LC-MS parameters for analytes and internal standards considered in method including precursor and product ions $(\mathrm{m} / \mathrm{z})$ and typical retention times.

Each batch included two replicates of quality control (QC) samples with three different concentrations; low QC $(0.3 \mu \mathrm{M})$, medium QC $(2.5 \mu \mathrm{M})$ and high QC $(3.8 \mu \mathrm{M})$. Usual acceptance criteria for each batch were that as least one QC at each concentration and four out of the six QCs were within 15\% of the theoretical concentration.

The method was validated using flavan-3-ol metabolite-free urine, spiked with the authentic standards E3'G, E3'S and 3Me5S to assess stability, specificity, matrix effects, precision and accuracy. The matrix effect was assessed by spiking flavan-3-ol metabolite-free urines from different sources with known amounts of analyte and internal standard and comparing their peak-area-ratio ratios. Method performance was assessed using data from quality control samples in 255 batches analyzed.

Human intervention studies. Three human intervention studies were conducted to investigate biomarker specificity, intake-response relationship and intra-individual variability. Details of the human intervention studies, with the exception of the investigation of intra-individual variability, have been described previously ${ }^{6}$. Briefly, the intervention consisted of fruit-flavoured non-dairy drinks that contained either specific flavan-3-ols (specificity study) or varying amounts of flavan-3-ols derived from cocoa (4 levels for the intake amount escalation study) and chocolate-flavored dairy drinks containing flavan-3-ols derived from cocoa (intra-variability study). The test materials were prepared freshly each day, and were matched regarding their macro- and micronutrient content and orosensory and physicochemical characteristics. All test materials were supplied by Mars, Incorporated (McLean, VA). A summary of the events taking during each study visit is summarized in Supplemental Fig. 3. These study protocols were approved by the Institutional Review Board of UC Davis. All participants gave their written informed consent to participate and all experiments were performed in accordance with local guidelines and regulations. These studies were registered as NCT03194620 and NCT03201822.

Study population. Healthy adults between 25 and 60 years of age (specificity study, Supplemental Fig. 4) and between 25 and 40 years of age (intake amount escalation study, Supplemental Fig. 5 ) were recruited by public advertisement in the city of Davis and surrounding areas (California, USA). Exclusion criteria were previously described as: body mass index (BMI) higher than $30 \mathrm{~kg} / \mathrm{m}^{2}$, blood pressure (BP) higher than 140/90 $\mathrm{mmHg}$, food allergies to test materials and a history of disease ${ }^{6}$. Throughout participation in the study (approximately, 8 weeks for the Specificity study, 4 weeks for the Intake escalation study and 2 weeks for the Intra-individual variability study), volunteers were asked to maintain their typical daily activities and diet. To control for potential dietary flavan-3-ol intake, volunteers were asked to follow a defined low-flavan-3-ol diet on the day prior to and during each study day, for a total of 2 days following a low-flavan-3-ol diet. All volunteers were instructed on how to follow a low-flavan-3-ol diet, receiving foods containing low or negligible amounts of flavanols including the dinner for the night previous to the study day. Volunteers were required to fast overnight ( $12 \mathrm{~h}$ water, ad libitum) before each study day.

Specificity study. In order to investigate the specificity of $\mathrm{SREM}_{\mathrm{B}}$ as nutritional biomarker of flavan-3-ol intake, we performed a randomized, double-masked and $8 \times$-crossover dietary intervention study with different possible precursors of structurally related $(-)$-epicatechin metabolites in healthy male adults $(n=12)$. Details of this study have been reported previously ${ }^{6}$. The flavan-3-ols tested were: (i) (-)-epicatechin, (ii) (-)-epigallocatechin, (iii) (-)-epicatechin-3-O-gallate, (iv) (-)-epigallocatechin-3-O-gallate (all compounds isolated from green tea, purity $>99 \%$ and food grade), (v) a 1:1:1: mixture of theaflavin-3-O-gallate, theaflavin-3'-O-gallate and theaflavin-3,3'-O-digallate, (vi) thearubigins (all isolated from black tea, purity $>95 \%$ and food grade) and (vii) procyanidin B-2 ((-)-epicatechin- $(4 \alpha 4 \beta)-(-)$-epicatechin; isolated from cocoa, purity $91 \%)$. One additional visit included the consumption of the test material without any flavan-3-ol added (control day). The amount of flavan3 -ols consumed per volunteer per study visit was equivalent to $34.8 \mathrm{mg}$ or $120 \mu \mathrm{mol}$, which is close to the mean intake amount of $(-)$-epicatechin consumed in the $\mathrm{UK}^{10}$. Test materials were consumed on a single occasion in the morning of the study visit. After intake, urine was collected over $24 \mathrm{~h}$ in two collection periods (from $0 \mathrm{~h}$ to $6 \mathrm{~h}$ post intake and $6 \mathrm{~h}$ to $24 \mathrm{~h}$ post intake), using a fresh container for each collection period with $20 \mathrm{~mL}$ of $2 \mathrm{M}$ sodium acetate $(\mathrm{pH}=4.5)$ and $2 \mathrm{~mL}$ of $0.5 \%(\mathrm{w} / \mathrm{v})$ thymol in isopropanol as preservatives. Volunteers returned containers upon completion of sample collection, and urine was stored at $-80^{\circ} \mathrm{C}$ until analysis. Volunteers were randomized to receive the 8 different test materials using computer-generated lists of random numbers via the randomly permuted block method. Study visits were separated by approximately 7 days for most volunteers, but never less than 3 days. The allocation list was generated by a researcher not involved with the recruitment and allocation of participants. Participants, study nurses, and researchers assessing outcomes as well as researchers involved with the statistical analysis of data were masked to the specific nature of intervention. The initial recruitment started in August 2016, and the study was conducted from August 2016 to January 2017. 
Intake escalation study. In order to investigate the association between flavan-3-ol intake and $\mathrm{SREM}_{\mathrm{B}}$ excre- $^{-}$ tion, we conducted a dietary intervention study where participants consumed different amounts of flavan-3-ol. Details of this study have been reported previously ${ }^{6}$. This was a randomized, double-masked study and followed a $4 \times$-crossover design, in which healthy male adults $(n=14)$ consumed four different amounts of flavan-3-ols that ranged from $95 \mathrm{mg}$ to $1424 \mathrm{mg}$, including $15 \mathrm{mg}$ to $227 \mathrm{mg}$ of $(-)$-epicatechin, which are amounts including and exceeding the range of intakes determined in the $\mathrm{UK}^{10}$. Test materials were consumed on a single occasion in the morning of the study visit. After intake, urine was collected over $24 \mathrm{~h}$ in 4 collection periods (from $0 \mathrm{~h}$ to $4 \mathrm{~h}$ post intake, $4 \mathrm{~h}$ to $8 \mathrm{~h}$ post intake, $8 \mathrm{~h}$ to $12 \mathrm{~h}$ post intake and $12 \mathrm{~h}$ to $24 \mathrm{~h}$ post intake), using a fresh container for each collection period with $20 \mathrm{~mL}$ of $2 \mathrm{M}$ sodium acetate $(\mathrm{pH}=4.5)$ and $2 \mathrm{~mL}$ of $0.5 \%(\mathrm{w} / \mathrm{v})$ thymol in isopropanol as preservatives. Volunteers returned the container upon completion of sample collection, and urine was stored at $-80^{\circ} \mathrm{C}$ until analysis. Volunteers were randomized to receive the four different test materials using computer-generated lists of random numbers via the randomly permuted block method. Study visits were separated by approximately 7 days for most volunteers, but never fewer than 3 days. The allocation list was generated by a researcher not involved with the recruitment and allocation of participants. Participants, study nurses, and researchers assessing outcomes as well as researchers involved with the statistical analysis of data were masked to the intervention. The initial recruitment started in April 2013, and the study was conducted from April to May 2013.

Intra-individual variability study. In order to investigate the intra-individual variation of $\mathrm{SREM}_{\mathrm{B}}$ excretion, we conducted a dietary intervention study where male and female participants consumed the same flavan-3-ol-containing drink on two different occasions separated by not less than 3 days (for study population see Supplemental Fig. 6). The amount of flavan-3-ols ingested was $542 \mathrm{mg}$, including $76 \mathrm{mg}$ of (-)-epicatechin, which is well within the range of intakes determined in the $\mathrm{UK}^{10}$. Test materials were consumed on a single occasion in the morning of the study visit. After intake, urine was collected over $24 \mathrm{~h}$ in 2 collection periods (from $0 \mathrm{~h}$ to $6 \mathrm{~h}$ post intake and $6 \mathrm{~h}$ to $24 \mathrm{~h}$ post intake), using a fresh container for each collection period with $20 \mathrm{~mL}$ of $2 \mathrm{M}$ sodium acetate $(\mathrm{pH}=4.5)$ and $2 \mathrm{~mL}$ of $0.5 \%(\mathrm{w} / \mathrm{v})$ thymol in isopropanol as preservatives. Volunteers returned the container upon completion of sample collection, and urine was stored at $-80^{\circ} \mathrm{C}$ until analysis. Study visits were separated by approximately 7 days for most volunteers, but never less than 3 days. The initial recruitment started in July 2018, and the study was conducted from August to September 2018.

Analytical method - human intervention studies. Urine samples from human intervention studies $(50 \mu \mathrm{L})$ were diluted with $100 \mu \mathrm{L}$ of $100 \mu \mathrm{M} 3$-methyl hippuric acid (internal standard) and analyzed by UPLC-MS within 24h, using a method described previously ${ }^{6}$. Samples were separated on a Waters Acquity UPLC (Waters, Milford, MA, USA) with an autosampler cooled at $5^{\circ} \mathrm{C}$. The injection volume was $37.5 \mu \mathrm{L}$. Chromatography was carried out at $25^{\circ} \mathrm{C}$ using a $100 \times 2.1 \mathrm{~mm}$ Kinetex C18 $1.7 \mu \mathrm{m}$, reversed phase column with a Krudcatcher (Phenomenex). The mobile phase, pumped at a flow rate of $0.5 \mathrm{~mL} / \mathrm{min}$, was (A) $5 \mathrm{mM}$ ammonium formate (pH 2.9); and (B) acetonitrile/methanol $(90: 10, \mathrm{v} / \mathrm{v})$. The gradient started with $5 \%$ B from $0-2 \mathrm{~min}$, then ramped to $17.5 \%$ from $2-3.5 \mathrm{~min}$, and then to $22.5 \%$ from 3.5-6 min. Detection was achieved with a Waters Micromass Quattro Premier spectrometer fitted with an electrospray interface (ESI; Waters). MS analysis was carried out in negative ionization mode by multiple reaction monitoring (MRM). Detection of (-)-epicatechin- $3^{\prime}$-glucuronide, $(-)$-epicatechin- $3^{\prime}$-sulfate, and $3^{\prime}$-O-methyl-(-)-epicatechin-5-sulfate utilized transitions from $464.5 \mathrm{~m} / \mathrm{z}$ to $289 \mathrm{~m} / \mathrm{z}, 368.8 \mathrm{~m} / \mathrm{z}$ to $289 \mathrm{~m} / \mathrm{z}$, and $382.5 \mathrm{~m} / \mathrm{z}$ to $302.5 \mathrm{~m} / \mathrm{z}$, respectively with dwell times of $0.05 \mathrm{~s}, 0.1 \mathrm{~s}$, and $0.1 \mathrm{~s}$; cone voltages of $25 \mathrm{~V}, 30 \mathrm{~V}$, and $35 \mathrm{~V}$; and collision energies of $18 \mathrm{~V}, 20 \mathrm{~V}$, and $18 \mathrm{~V}$ respectively. Data processing was performed using Masslynx software (Waters). MS conditions were set by tuning with the corresponding compound. Source and desolvation temperature were set at $150{ }^{\circ} \mathrm{C}$ and $500^{\circ} \mathrm{C}$, respectively, while desolvation and cone gas were set at $900 \mathrm{~L} / \mathrm{h}$ and $30 \mathrm{~L} / \mathrm{h}$, respectively. In both ionization modes, capillary, cone, and lens voltage were set at $4 \mathrm{kV}, 30 \mathrm{~V}$, and $1 \mathrm{~V}$, respectively. The identification and quantification of the compounds were based on co-elution with authentic standards. The precision and accuracy of the method was better than $5 \%$ and $20 \%$, respectively.

Cohort studies. We have investigated the feasibility of $\mathrm{SREM}_{\mathrm{B}}$ as nutritional biomarker of (-)-epicatechin in samples from two cohort studies, EPIC Norfolk and Challenge.

EPIC Norfolk. 25,639 apparently healthy men and women between 40 and 75 years of age, living in and around Norwich, UK, were recruited between 1993 and $1997^{11}$. Spot urine samples were collected during the baseline health examination and stored at $-20^{\circ} \mathrm{C} ; 24,341$ spot urine samples were available for analysis. The study was approved by the Norwich District Health Authority Ethics Committee, and all participants gave signed informed consent.

Challenge study. 158 cognitively normal elderly people ((77 women, $81 \mathrm{men})$ aged 60 to 91 years old) living in and around Oxford were recruited in 1997 as part of the OPTIMA (Oxford Project to Investigate Memory and Ageing) study ${ }^{12} .24 \mathrm{~h}$ urine samples were collected at baseline and stored at $-70^{\circ} \mathrm{C} ; 145$ samples were available for analysis. The Central Oxford Research Ethics Committee granted ethical approval. Informed consent was obtained from all participants for all testing.

Statistical analyses. Associations between biomarker and intake were assessed with linear regression models using R 3.5. $0^{13}$ with the boot ${ }^{14}$, ggplot $2^{15}$ and $r m s^{16}$ packages. Differences between epicatechin precursors where investigated using one-way ANOVA with Tukey's Honest Significant Difference method ${ }^{17}$. 


\begin{tabular}{|c|c|c|c|c|c|c|c|c|c|}
\hline & \multicolumn{3}{|l|}{$\begin{array}{l}\text { Low QC } \\
0.3 \mu \mathrm{M}\end{array}$} & \multicolumn{3}{|c|}{$\begin{array}{l}\text { Medium QC } \\
2.5 \mu \mathrm{M}\end{array}$} & \multicolumn{3}{|l|}{$\begin{array}{l}\text { High QC } \\
3.8 \mu \mathrm{M}\end{array}$} \\
\hline & Mean (SD) & $\% \mathrm{CV}$ & $\%$ RE & $\begin{array}{l}\text { Mean } \\
\text { (SD) }\end{array}$ & $\% \mathrm{CV}$ & $\%$ RE & $\begin{array}{l}\text { Mean } \\
\text { (SD) }\end{array}$ & $\% \mathrm{CV}$ & $\%$ RE \\
\hline (-)-epicatechin-3'-glucuronide & $0.32(0.03)$ & 10.0 & 6.4 & $2.6(0.3)$ & 9.4 & 4.5 & $3.9(0.3)$ & 7.5 & 1.9 \\
\hline$(-)$-epicatechin- $3^{\prime}$-sulfate & $0.32(0.03)$ & 9.3 & 5.4 & $2.6(0.2)$ & 8.5 & 2.7 & $3.8(0.3)$ & 7.7 & 1.1 \\
\hline 3'-O-methyl-(-)-epicatechin-5-sulfate & $0.32(0.03)$ & 10.4 & 6.4 & $2.6(0.2)$ & 8.8 & 4.1 & $3.9(0.3)$ & 8.6 & 1.4 \\
\hline
\end{tabular}

Table 3. Long term precision (\%CV) and accuracy (\%RE, difference of mean calculated concentration and nominal concentration, standardized by nominal concentration) data for the three SREMs used as biomarker $\left(\mathrm{SREM}_{\mathrm{B}}\right)$, based on 575 samples (except (-)-epicatechin-3'-sulfate high QC for which 370 samples only were available).

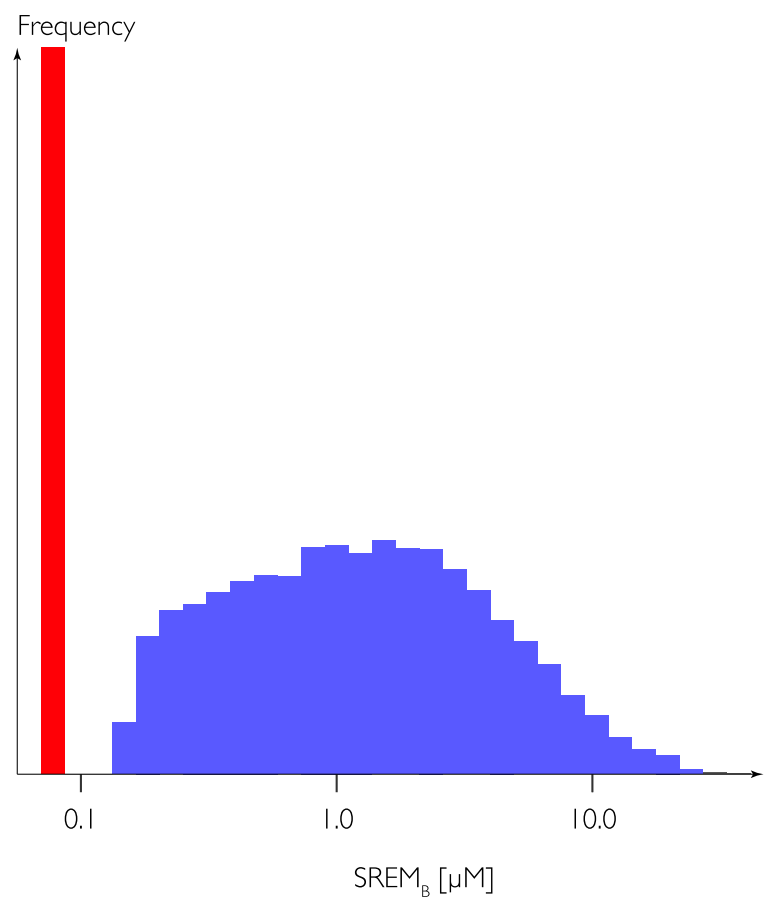

Figure 1. Histogram of urinary $\operatorname{SREM}_{B}$ concentration in spot urines of 24,341 participants of EPIC Norfolk. The lower limit of quantification was $0.1 \mu \mathrm{M}$.

\section{Results}

Analytical method validation and performance. The analysis of nutritional biomarker requires robust and validated analytical methods. We have included the sum of three SREMs $((-)$-epicatechin-3'-glucuronide $\left(\mathrm{E}^{\prime} \mathrm{G}\right),(-)$-epicatechin-3'-sulfate (E3'S) and 3'-O-methyl-(-)-epicatechin-5-sulfate (MeE5S), SREM $\left.{ }_{\mathrm{B}}\right)$ in an LC-MS method developed for the analysis of gVLM (5-( $3^{\prime}, 4^{\prime}$-dihydroxyphenyl)- $\gamma$-valerolactone- $3^{\prime} / 4^{\prime}-\mathrm{O}$-glucuronide (gVL34G) and 5-( $3^{\prime}, 4^{\prime}$-dihydroxyphenyl)- $\gamma$-valerolactone- $3^{\prime} / 4^{\prime}$-sulfate (gVL34S)) as biomarker of flavan-3-ol intake, as this method can be automated and is suitable for high-throughput analysis ${ }^{6}$. The precision and accuracy of the method was well within the usual GLP criteria of $15 \%$ and better than $10 \%$ for most compounds. The estimated limit of detection (signal-to-noise ratio better than 5 -to-1) was at least $0.1 \mu \mathrm{M}$ for all compounds. Where matrix effects were evident, these were adequately compensated for by the use of an isotope-labelled internal standard. All compounds were tested for their stability in urine under different conditions, including at high temperature $\left(6 \mathrm{~h}\right.$ at $37^{\circ} \mathrm{C}, 24 \mathrm{~h}$ at room temperature) and following four freeze-thaw cycles $\left(-80^{\circ} \mathrm{C}\right)$, and the extracted samples were tested for $72 \mathrm{~h}$ at $5{ }^{\circ} \mathrm{C}$ and seven days at $-20^{\circ} \mathrm{C}$. All compounds were stable under these conditions and showed no indication of deterioration and we did not observe any batch effect or long-term drift of results. Table 3 shows long term precision and accuracy data for the three (-)-epicatechin metabolites $\left(\mathrm{SREM}_{\mathrm{B}}\right)$.

In an analysis of spot urines collected from 24,341 participants of EPIC Norfolk, biomarker concentrations above the limit of quantification $(0.1 \mu \mathrm{mol} / \mathrm{L})$ were found in 20,277 participants $(83 \%)$. Figure 1 shows the distribution of SREM $_{B}$ concentrations, which was skewed to the left with a median (IQR) of $1.2(0.5-2.9) \mu \mathrm{mol} / \mathrm{L}$ and a mean (SD) of $2.4(3.2) \mu \mathrm{mol} / \mathrm{L}$.

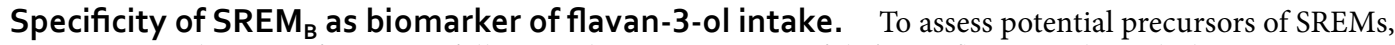
we investigated $\mathrm{SREM}_{\mathrm{B}}$ formation following the consumption of different flavan-3-ols, including monomeric flavan-3-ols, oligomeric (procyanidins) and galloylated flavan-3-ols in male adults $(n=12)$. Figure 2 shows that 


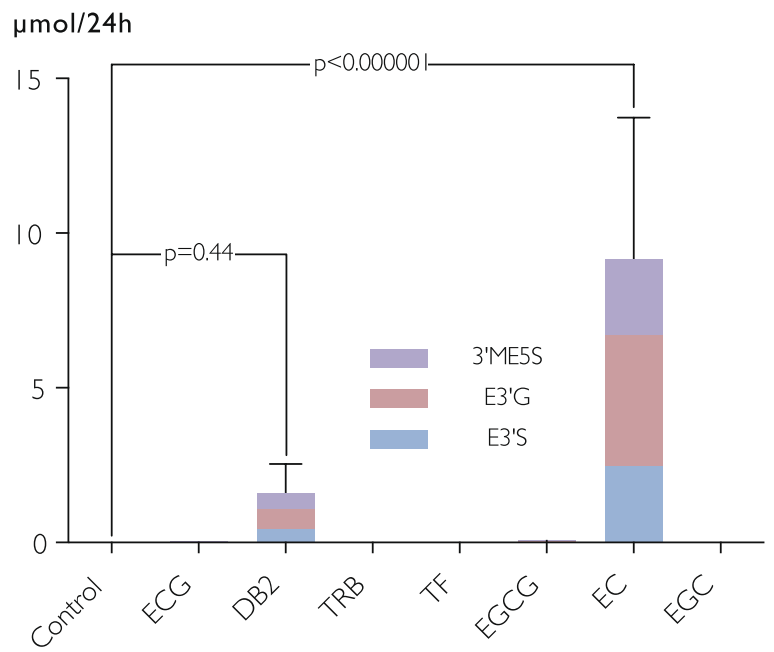

Figure 2. Urinary excretion of structurally related (-)-epicatechin metabolites $\left(\mathrm{SREM}_{\mathrm{B}}\right)$ following the consumption of different flavan-3-ols in male adults $(n=12)$. ECG, $(-)$-epicatechin-3-O-gallate; DB2, procyanidin dimer B2; TRB: thearubigins; TF: theaflavins; EGCG, (-)-epigallocatechin-3-O-gallate; EC, (-)-epigallocatechin; EGC, (-)-epigallocatechin. Differences in excretion were compared using a one-way ANOVA and a pair-wise comparison with Tukey's Honest Significant Difference method ${ }^{17}$.

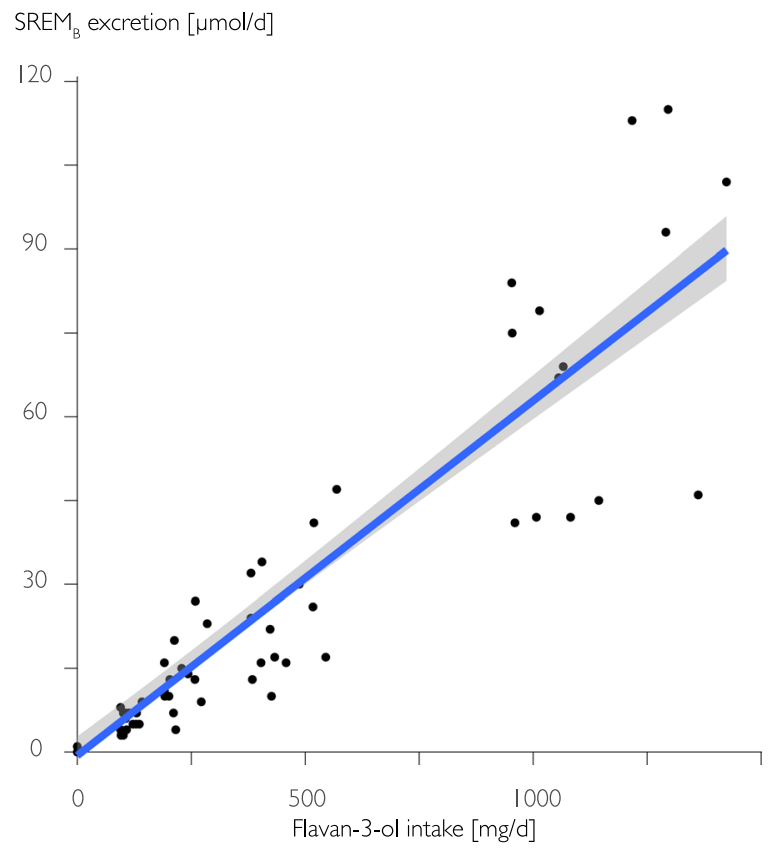

Figure 3. Association between flavan-3-ol intake and biomarker $\left(\operatorname{SREM}_{\mathrm{B}}\right)$ excretion in male adults $(\mathrm{n}=14)$. The data show a strong correlation between intake and biomarker $\left(\mathrm{R}^{2}: 0.86\right.$ (95\% CI 0.81; 0.92)).

only the consumption of (-)-epicatechin resulted in statistically significant increase of $\mathrm{SREM}_{\mathrm{B}}$ excreted in urine. While the intake of procyanidin dimer B2 did not statistically increase $\mathrm{SREM}_{\mathrm{B}}$ excreted in urine $(\mathrm{p}=0.4$; one-way ANOVA), the contribution could be estimated as $2 \%$ of the amount of procyanidin dimer B2 ingested. The rest of the flavan-3-ols ingested, including (-)-epicatechin-3-O-galate, did not contribute to the presence of $S_{R E M}$ excreted in urine.

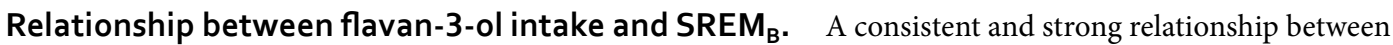
flavan-3-ol intake and $\mathrm{SREM}_{\mathrm{B}}$ excretion in $24 \mathrm{~h}$ urine is crucial to establish its suitability as biomarker. We have therefore investigated this association with an intake-escalation study in healthy male adults $(\mathrm{n}=14)$ with intakes of up to $1400 \mathrm{mg} / \mathrm{d}$ of flavan-3-ols $(230 \mathrm{mg} / \mathrm{d}$ of (-)-epicatechin), which exceeds the range of likely dietary intake ${ }^{10,18}$. The results show a strong correlation between intake and $\operatorname{SREM}_{B}\left(\mathrm{R}^{2}: 0.86\right.$ (95\% CI 0.81; 0.92), Spearman's rank correlation: $\rho=0.96$; Pearson's $r=0.93$; Fig. 3 ). These results were only slightly attenuated when 
excluding high intakes above $750 \mathrm{mg} / \mathrm{d}\left(\mathrm{R}^{2}: 0.82\right.$ (95\% CI 0.73; 0.88), Spearman's rank correlation: $\rho=0.94$; Pearson's $\mathrm{r}=0.90)$. We also investigated the extent of the relationship between $(-)$-epicatechin intake and $\mathrm{SREM}_{\mathrm{B}}$. The results show that the relationship between dietary intake of (-)-epicatechin and $\mathrm{SREM}_{\mathrm{B}}$ is $\beta=0.12$ $(95 \%$ CI $0.11 ; 0.12)$ with a median recovery of $10 \%$ (IQR $7-13 \%)$.

Intra- and inter-individual variability. Intra- and inter-individual variability of the association between flavan-3-ol intake and $\mathrm{SREM}_{\mathrm{B}}$ affect its suitability as biomarker of intake. We have therefore investigated intra- and inter-individual variability in men and women $(\mathrm{n}=15, \mathrm{~m} / \mathrm{f}: \mathrm{m} / \mathrm{f})$ consuming $543 \mathrm{mg}$ of flavan-3-ols (including $263 \mu \mathrm{mol}(-)$ epicatechin) on two occasions. Mean $\left( \pm\right.$ SEM) SREM $_{B}$ excretion at the first and second intervention was $46.2 \pm 4.2 \mu \mathrm{mol}$ and $42.8 \pm 4.4 \mu \mathrm{mol}$ respectively, with no statistically significant difference (paired t-test, $\mathrm{p}=0.2$ ). There was a strong correlation between $\mathrm{SREM}_{\mathrm{B}}$ excretion at first and second visit (Pearson's $\left.\rho: 0.83 ; \mathrm{R}^{2}=0.66\right)$. The intra-individual variability was $12 \pm 2.5 \%$, with only two participants having differences larger than $20 \%$.

Applicability of the candidate biomarker to estimate intake in free living individuals (Challenge study). We analyzed $24 \mathrm{~h}$ urine $(\mathrm{n}=158)$ collected in the context of the Challenge study. With a median recovery of $10 \%$ as found in the intake-escalation study, the estimated intake of (-)-epicatechin was of $14.0 \pm 1 \mathrm{mg} / \mathrm{d}$, ranging from 0 to $58 \mathrm{mg} / \mathrm{d}$.

\section{Discussion}

In this series of studies, we have investigated the suitability of three structurally related (-)-epicatechin metabolites $\left((-)\right.$-epicatechin- $3^{\prime}$-glucuronide $\left(E 3^{\prime} G\right),(-)$-epicatechin- $3^{\prime}$-sulfate $\left(E 3^{\prime} S\right)$ and $3^{\prime}$-O-methyl$(-)$-epicatechin-5-sulfate $\left.(\mathrm{MeE} 5 \mathrm{~S}), \mathrm{SREM}_{\mathrm{B}}\right)$ as nutritional biomarkers of dietary (-)-epicatechin intake using criteria by IARC ${ }^{19}$ and the Institute of Medicine ${ }^{20}$ as described previously ${ }^{6}$. Here, we discuss whether or not $\mathrm{SREM}_{\mathrm{B}}$ is suitable as biomarker of flavan-3-ol intake:

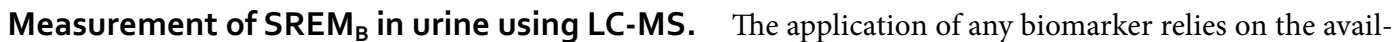
ability of accurate, precise and robust analytical methods, which ideally should be suitable for automation and high-throughput analysis to allow their application to large studies. We have developed an analytical method which is suitable for the rapid analysis of the two main types of flavan-3-ol metabolites, structurally related (-)-epicatechin metabolites (SREM) and metabolites of the microbial fission products of flavan-3-ols, $\gamma$-valerolactones ${ }^{6}$. This method allows us to determine a range of different metabolites in a short time, and therefore is suitable for the analysis of samples even from large studies. The accurate and precise analysis of these metabolites requires the availability of authentic standards, as other methods, relying for example on enzymatic deconjugation ${ }^{21,22}$ or the identification of metabolites using their tandem-MS fragmentation pattern and quantification as aglycone ${ }^{23}$, considerable bias. The measurement error introduced by these methods is dependent on metabolite and metabolite concentration, and therefore does not only affect absolute but also relative quantification. This makes such methods unsuitable to rank participants according to intake, as is common practice for many nutritional biomarkers. We have confirmed the performance of the method following generally accepted standards ${ }^{24}$, with precision and accuracy well below $15 \%$ for the entire concentration range. In combination with a run time of eight minutes and the suitability for robotic automation, this makes the method suitable for the high-throughput analysis of large-scale studies. The application of the method to two different cohort studies in the UK shows that the validation range is sufficient to measure $\mathrm{SREM}_{\mathrm{B}}$ and $\mathrm{gVLM}^{23}$ in the majority of participants.

Dietary precursors of SREM . The importance of the specificity of nutritional biomarkers has been discussed previously ${ }^{5,19}$, but in contrast to the relationship between nutritional biomarker and intake, this is not often assessed. We have shown that (-)-epicatechin, but none of the other flavan-3-ols tested, resulted in the formation of $\mathrm{SREM}_{\mathrm{B}}$. This confirms results from our previous studies ${ }^{3,25}$, showing that oligo- and polymeric procyanidins are not cleaved into their flavan-3-ol subunits.

The data therefore show that urinary $\operatorname{SREM}_{\mathrm{B}}$ is specific for (-)-epicatechin, but considering that this compound could be present in its enantiomeric form $(+)$-epicatechin, it is worth questioning whether $\mathrm{SREM}_{\mathrm{B}}$ could be specific to assess (-)-epicatechin intake. In this context, the biosynthetic pathways of flavonoids in plants result predominantly in (-)-epicatechin ${ }^{26}$ and only small amounts of $(+)$-epicatechin are found in the diet ${ }^{27}$ and thus not relevant as a contributor to $\mathrm{SREM}_{\mathrm{B}}$. Furthermore, previous data show that (+)-epicatechin intake does not significantly contribute to epicatechin-3'- $\beta$-glucuronide, one of the main contributors to the set of metabolites included in $\mathrm{SREM}_{\mathrm{B}}{ }^{28}$. Thus, $\mathrm{SREM}_{\mathrm{B}}$ will predominantly reflect the intake of (-)-epicatechin.

Association between dietary intake and SREM $\mathrm{B}_{\mathrm{B}}$. We have shown a statistically significant linear association between (-)-epicatechin and $\mathrm{SREM}_{B}$ excretion in $24 \mathrm{~h}$ urine in an intake-escalation study $\left(\mathrm{R}^{2}: 0.86(95 \%\right.$ CI $0.81 ; 0.92)$ ). Importantly, the association of individual metabolites showed a weaker association with intake than did the sum $\left(\mathrm{SREM}_{\mathrm{B}}\right)$. We demonstrated that this is due to changes in the relative contribution of different $\mathrm{SREM}_{\mathrm{B}}$ metabolites according to intake amount. This observation is not novel and concurs with previous studies demonstrating a change in sulfation/glucuronidation ratio with the amount of phenolic compounds ingested ${ }^{29}$. Thus, these results show that it is important to use a combination of different metabolites as biomarkers of intake, as changes in metabolism due to intake could result in mis-estimations of intake. The combination of metabolites permits both, improving the strength of the association and increasing the recovery assessed for (-)-epicatechin. Overall, the data presented here show that changes in urinary excretion of SREM $\mathrm{B}_{\mathrm{B}}$ reflect changes in dietary intake of (-)-epicatechin consistently and are therefore prognostic of intake. 


\begin{tabular}{|l|l|l|}
\hline & gVLM & SREM $_{\mathbf{B}}$ \\
\hline Sample & 24h urine, spot urine & 24h urine \\
\hline Metabolites included & gVL3'S, gVL3'G, gVL4'G $^{\prime}$ & $\begin{array}{l}\text { E3'S, E3'G, } \\
3^{\prime} \mathrm{Me} 5 \mathrm{~S}\end{array}$ \\
\hline Specificity & $\begin{array}{l}\text { Flavan-3-ol monomers, } \\
\text { ECG, procyanidins }\end{array}$ & (-)-epicatechin \\
\hline Type of biomarker & Concentration/surrogate & recovery \\
\hline
\end{tabular}

Table 4. Comparison of gVLM and $\mathrm{SREM}_{\mathrm{B}}$ as biomarkers.

Suitability of $S_{R E M}$ as nutritional biomarker of (-)-epicatechin intake. Our results show that $24 \mathrm{~h}$ urinary $S_{\mathrm{SEM}} \mathrm{B}_{\mathrm{B}}$ excretion meets the criteria set to evaluate candidate nutritional biomarkers. Furthermore and according to the criterion for biomarker evaluation used previously by the NPAAS-FS (Nutrition and Physical Activity Assessment Study Feeding Study; $\left.\mathrm{R}^{2} \geq 0.36\right)^{30,31}$, the data also suggest that $24 \mathrm{~h}$ excretion of $\mathrm{SREM}_{\mathrm{B}}$ can be used as recovery biomarker of (-)-epicatechin and therefore used to estimate actual (-)-epicatechin intake. The correlation between intake and biomarker is $>0.8$ and therefore meets the criteria commonly used for recovery biomarkers ${ }^{5}$. Furthermore, the data available from this and previous studies, in particular the pharmacokinetic data ${ }^{32}$, provide precise and quantitative knowledge of the physiological balance between intake and output which is required for recovery biomarkers ${ }^{33}$. In comparison with urinary nitrogen as biomarker of dietary protein intake, one of the best characterized recovery biomarkers, the correlations between intake and biomarker are comparable $\left(0.8-0.9\right.$ for nitrogen as a biomarker of dietary protein ${ }^{34}, 0.9$ for urinary $\mathrm{SREM}_{\mathrm{B}}$ as biomarker of epicatechin intake). Although the recovery of (-)-epicatechin as urinary $\operatorname{SREM}_{B}(10 \% \pm 1 \%)$ was lower than the recovery of dietary nitrogen in urine $\left(91 \% \pm 1 \%{ }^{34}\right)$, the variability of recovery was similar and most notably the recovery was neither skewed nor dependent on intake. When applying this biomarker to the $24 \mathrm{~h}$ urine samples collected in Challenge, the estimated intake of (-)-epicatechin was $14.0 \pm 1 \mathrm{mg} / \mathrm{d}$, ranging from 0 to $58 \mathrm{mg} / \mathrm{d}$.

Comparison with $\gamma$-valerolactone metabolites (gVLM). We have previously evaluated gVLM as biomarker of flavan-3-ol intake and shown that, due to its plasma half-life of approximately $6 \mathrm{~h}^{32}$ and the frequent consumption of flavan-3-ol containing foods, it can be used as surrogate ${ }^{35}$ or concentration biomarker both in $24 \mathrm{~h}$ urine and spot urine samples ${ }^{6}$. However, the large inter-individual variability in colonic microbial metabolism makes gVLM not suitable as recovery biomarker. Conversely, the short plasma half-life of $\mathrm{SREM}_{\mathrm{B}}$, approximately $2 \mathrm{~h}$, assures a complete excretion within $24 \mathrm{~h}$ of urine collection that, added up to the high association with intake, makes $\mathrm{SREM}_{\mathrm{B}}$ ideal for a recovery biomarker. Due to the different flavanols contributing to gVLM and $\mathrm{SREM}_{\mathrm{B}}$ and differences in half-life, the measurement of both biomarkers could provide complementary information. In $24 \mathrm{~h}$ urine samples, a combination of $\mathrm{SREM}_{\mathrm{B}}$ and $\mathrm{gVLM}$ could be used to differentiate between $(-)$-epicatechin and total flavan-3-ol intake, whereas in spot urine samples, $\mathrm{SREM}_{\mathrm{B}}$ can be used as marker of very short-term, acute (-)-epicatechin intake (see Table 4 for a direct comparison).

Strengths and limitations. This study has a number of strengths, but also some limitations which need to be considered when interpreting results. A key strength of the study is the carefully validated analytical method and the availability of authentic standards for all three (-)-epicatechin metabolites, and we have shown previously that this is a major contributor to the accuracy of polyphenol metabolite quantification by LC-MS ${ }^{23}$. The reliance on a single deuterium-labelled internal standard $\left(\mathrm{D}_{2} / \mathrm{D}_{3}-\mathrm{E} 3^{\prime} \mathrm{G}\right)$ for quantification of $\mathrm{E} 3^{\prime} \mathrm{G}, \mathrm{E} 3^{\prime} \mathrm{S}$ and $3^{\prime} \mathrm{Me} 5 \mathrm{~S}$ might have affected quantification of the latter two metabolites, but the method validation showed very good precision and accuracy. A further strength of the study is the careful investigation of the intake response relationship and in particular the specificity of $\mathrm{SREM}_{\mathrm{B}}$. Providing the flavan-3-ol intervention on a single occasion using a defined test material was different from what could be expected as part of the habitual diet, but permitted a tight control on the amounts and type of flavan-3-ols consumed during the intervention and thus, obtain essential information on the specificity and intake-amount relationship of SREM $\mathrm{B}_{\mathrm{B}}$ as biomarkers of (-)-epicatechin intake. A limitation of the study is the reliance on two UK cohorts to evaluate the applicability of $\mathrm{SREM}_{\mathrm{B}}$ as urinary biomarker. While flavan-3-ol intake in the UK is generally higher than in many other countries, their main source is tea ${ }^{10,18}$ with a very different flavan-3-ol composition than for example pome fruits. While this ensured that there was a wide range of flavanol intake within the study population, it could have affected outcomes. It is therefore important to apply this biomarker to studies from countries with more diverse sources of flavanols. Finally, it should be noted that the criteria chosen for the evaluation of $\mathrm{SREM}_{\mathrm{B}}$ have not been specifically created for nutritional biomarkers of dietary bioactives. The criteria used were derived from the frameworks provided by IARC and IOM. Future discussions, such as suggested previously ${ }^{36}$, are crucial to develop specific criteria for the evaluation of nutritional biomarkers, and thus foster the development of new biomarkers of dietary bioactives for the replacement of biased methods for intake assessment.

\section{Conclusion}

In our studies, we have shown that $24 \mathrm{~h}$ urinary excretion of a combination of three structurally related $(-)$-epicatechin metabolites $\left((-)\right.$-epicatechin- ${ }^{\prime}$-glucuronide $\left(\mathrm{E} 3^{\prime} \mathrm{G}\right),(-)$-epicatechin-3'-sulfate $\left(\mathrm{E} 3^{\prime} \mathrm{S}\right)$ and 3'-O-methyl-(-)-epicatechin-5-sulfate $\left.(\mathrm{MeE} 5 \mathrm{~S}), \mathrm{SREM}_{\mathrm{B}}\right)$ meet the criteria for a recovery biomarker in $24 \mathrm{~h}$ urines and can therefore be used as a nutritional biomarker to estimate actual (-)-epicatechin intake. The method we have developed is suitable for automation and therefore high-throughput analysis, and we have applied it successfully to more than 24,000 samples of the EPIC Norfolk cohort. The development of this biomarker would not have been possible without the availability of authentic standards and detailed pharmacokinetic data. 
We have shown that $\mathrm{SREM}_{\mathrm{B}}$ is specific for (-)-epicatechin and not any other flavan-3-ol commonly consumed. Our findings provide an accurate method for the objective estimation of dietary (-)-epicatechin, the most likely bioactive flavan-3-ol. This opens up the possibility to investigate associations between $(-)$-epicatechin intake and health outcomes in large-scale observational studies. In the future this method could hold the potential to objectively assess dietary epicatechin adequacy as part of approaches for personalised nutrition goals.

\section{References}

1. Schroeter, H. et al. Recommending flavanols and procyanidins for cardiovascular health: Current knowledge and future needs. Mol. Aspects Med. 31, 546-557 (2010).

2. Recommending flavanols and procyanidins for cardiovascular health: Revisited. Mol. Aspects Med. 61, 63-75 (2018).

3. Rodriguez-Mateos, A. et al. Assessing the respective contributions of dietary flavanol monomers and procyanidins in mediating cardiovascular effects in humans: randomized, controlled, double-masked intervention trial. Am J Clin Nutr 31, 546-1237 (2018).

4. Kuhnle, G. G. C. Nutritional biomarkers for objective dietary assessment. J. Sci. Food Agric. 92, 1145-1149 (2012).

5. Jenab, M., Slimani, N., Bictash, M., Ferrari, P. \& Bingham, S. A. Biomarkers in nutritional epidemiology: applications, needs and new horizons. Hum. Genet. 125, 507-525 (2009).

6. Ottaviani, J. I. et al. Evaluation at scale of microbiome-derived metabolites as biomarker of flavan-3-ol intake in epidemiological studies. Sci Rep 8, 9859 (2018).

7. Sun, Q. et al. Urinary Excretion of Select Dietary Polyphenol Metabolites Is Associated with a Lower Risk of Type 2 Diabetes in Proximate but Not Remote Follow-Up in a Prospective Investigation in 2 Cohorts of US Women. Journal of Nutrition 145, 1280-1288 (2015).

8. Zhang, M., Erik Jagdmann, G. Jr., Van Zandt, M., Beckett, P. \& Schroeter, H. Enantioselective synthesis of orthogonally protected (2R,3R)-(-)-epicatechin derivatives, key intermediates in the de novo chemical synthesis of (-)-epicatechin glucuronides and sulfates. Tetrahedron: Asymmetry 24, 362-373 (2013).

9. Zhang, M. et al. Chemical Synthesis and Characterization of Epicatechin Glucuronides and Sulfates: Bioanalytical Standards for Epicatechin Metabolite Identification. J Nat Prod 76, 157-169 (2013).

10. Vogiatzoglou, A. et al. Assessment of the dietary intake of total flavan-3-ols, monomeric flavan-3-ols, proanthocyanidins and theaflavins in the European Union. Br J Nutr 111, 1463-1473 (2014).

11. Day, N. et al. EPIC-Norfolk: study design and characteristics of the cohort. European Prospective Investigation of Cancer. $\mathrm{Br} J$ Cancer 80(Suppl 1), 95-103 (1999).

12. Budge, M. M., de Jager, C., Hogervorst, E. \& Smith, A. D. Total plasma homocysteine, age, systolic blood pressure, and cognitive performance in older people. J Am Geriatr Soc 50, 2014-2018 (2002).

13. R Core Team. R: A Language and Environment for Statistical Computing. 1 (2018).

14. Canty, A. \& Ripley, B. boot: Bootstrap R (S-Plus) Functions. (2017).

15. Wickham, H. ggplot2: Elegant Graphics for Data Analysis. (Springer-Verlag New York, 2009).

16. Harrell, F. E. Jr. rms: Regression Modeling Strategies (2017).

17. Tukey, J. W. Comparing individual means in the analysis of variance. Biometrics 5, 99-114 (1949).

18. Vogiatzoglou, A. et al. Flavonoid intake in European adults (18 to 64 Years). PLoS One 10 (2015).

19. Kaaks, R., Riboli, E. \& Sinha, R. Biochemical markers of dietary intake. IARC Sci Publ 103-126 (1997).

20. The National Academies of Sciences, Engineering and Medicine, Micheel, C. M. \& Ball, J. R. Evaluation of Biomarkers and Surrogate Endpoints in Chronic Disease., https://doi.org/10.17226/12869 (National Academies Press (US), 2010).

21. Saha, S. et al. Human O-sulfated metabolites of (-)-epicatechin and methyl-(-)-epicatechin are poor substrates for commercial aryl-sulfatases: Implications for studies concerned with quantifying epicatechin bioavailability. Pharmacological Research 65, 592-602 (2012).

22. Ottaviani, J. I., Momma, T. Y., Kuhnle, G. G., Keen, C. L. \& Schroeter, H. Structurally related (-)-epicatechin metabolites in humans: assessment using de novo chemically synthesized authentic standards. Free Radic Biol Med 52, 1403-1412 (2012).

23. Ottaviani, J. I., Fong, R. Y., Borges, G., Schroeter, H. \& Crozier, A. Use of LC-MS for the quantitative analysis of (poly)phenol metabolites does not necessarily yield accurate results: Implications for assessing existing data and conducting future research. Free Radic Biol Med 124, 97-103 (2018).

24. Food and Drug Administration. Guidance for Industry - Bioanalytical Method Validation. Tobacco Smoke Exposure Biomarkers 247-264, https://doi.org/10.1201/b18276-17 (2001).

25. Ottaviani, J. I., Kwik-Uribe, C., Keen, C. L. \& Schroeter, H. Intake of dietary procyanidins does not contribute to the pool of circulating flavanols in humans. 95, 851-858 (2012).

26. Kofink, M., Papagiannopoulos, M. \& Galensa, R. Enantioseparation of catechin and epicatechin in plant food by chiral capillary electrophoresis. Eur Food Res Technol 225, 569-577 (2006).

27. Alanon, M. E., Castle, S. M., Siswanto, P. J., Cifuentes-Gómez, T. \& Spencer, J. P. E. Assessment of flavanol stereoisomers and caffeine and theobromine content in commercial chocolates. Food Chemistry 208, 177-184 (2016).

28. Ottaviani, J. I. et al. The stereochemical configuration of flavanols influences the level and metabolism of flavanols in humans and their biological activity in vivo. Free Radic Biol Med 50, 237-244 (2011).

29. Koster, H., Halsema, I., Scholtens, E., Knippers, M. \& Mulder, G. J. Dose-dependent shifts in the sulfation and glucuronidation of phenolic compounds in the rat in vivo and in isolated hepatocytes. The role of saturation of phenolsulfotransferase. Biochem Pharmacol 30, 2569-2575 (1981).

30. Lampe, J. W. et al. Dietary biomarker evaluation in a controlled feeding study in women from the Women's Health Initiative cohort. Am J Clin Nutr 105, 466-475 (2017).

31. Yun, H. Y. et al. Serum nitrogen and carbon stable isotope ratios meet biomarker criteria for fish and animal protein intake in a controlled feeding study of a Women's Health Initiative cohort. Journal of Nutrition (2018).

32. Ottaviani, J. I. et al. The metabolome of [2-14C](-)-epicatechin in humans: implications for the assessment of efficacy, safety, and mechanisms of action of polyphenolic bioactives. Sci Rep 6, 1-10 (2016).

33. Kaaks, R., Ferrari, P., Ciampi, A., Plummer, M. \& Riboli, E. Uses and limitations of statistical accounting for random error correlations, in the validation of dietary questionnaire assessments. Public Health Nutr 5, 969-976 (2002).

34. Bingham, S. A. et al. Validation of weighed records and other methods of dietary assessment using the $24 \mathrm{~h}$ urine nitrogen technique and other biological markers. Br J Nutr 73, 531-550 (1995)

35. Keogh, R. H., White, I. R. \& Bingham, S. A. Using surrogate biomarkers to improve measurement error models in nutritional epidemiology. Stat Med 32, 3838-3861 (2013).

36. Praticò, G. et al. Guidelines for Biomarker of Food Intake Reviews (BFIRev): how to conduct an extensive literature search for biomarker of food intake discovery. Genes Nutr 13, 3 (2018).

37. Rothwell, J. A. et al. Phenol-Explorer 3.0: a major update of the Phenol-Explorer database to incorporate data on the effects of food processing on polyphenol content. Database 2013, bat070-bat070 (2013). 


\section{Acknowledgements}

We thank all EPIC-Norfolk study participants and staff for their contribution to the study. We thank all volunteers at UC Davis. NGF acknowledges MRC Epidemiology Unit core support [MC_UU_12015/5] and NIHR Biomedical Research Centre Cambridge: Nutrition, Diet and Lifestyle Research Theme [IS-BRC-1215-20014].

\section{Author Contributions}

J.I.O., H.S. and G.G.C.K. designed the research. J.I.O., R.F., J.K. and J.L.E. conducted the intake escalation and specificity studies. J.I.O., R.F., P.B.G., D.H.M., A.T., A.W. and G.G.C.K. developed and validated the analytical methods. A.B., N.G., A.V., D.L., P.B.G., D.H.M., A.T., A.W., R.L. and G.G.C.K. analysed and processed samples of the prospective cohort study. K.T.K. and N.J.W. are PIs of EPIC Norfolk, A.D.S. is PI of OPTIMA; N.G.F. provided intellectual input and edited the manuscript. J.I.O., H.S. and G.G.C.K. had primary responsibility for the final content. All authors read and approved the final manuscript.

\section{Additional Information}

Supplementary information accompanies this paper at https://doi.org/10.1038/s41598-019-49702-z.

Competing Interests: H.S. and J.I.O. are employed by Mars, Inc., a company engaged in flavanol research and flavanol-related commercial activities. G.G.C.K. has received an unrestricted research grant from Mars Inc. None of the other authors has a conflict of interest to declare.

Publisher's note: Springer Nature remains neutral with regard to jurisdictional claims in published maps and institutional affiliations.

Open Access This article is licensed under a Creative Commons Attribution 4.0 International ense, which permits use, sharing, adaptation, distribution and reproduction in any medium or format, as long as you give appropriate credit to the original author(s) and the source, provide a link to the Creative Commons license, and indicate if changes were made. The images or other third party material in this article are included in the article's Creative Commons license, unless indicated otherwise in a credit line to the material. If material is not included in the article's Creative Commons license and your intended use is not permitted by statutory regulation or exceeds the permitted use, you will need to obtain permission directly from the copyright holder. To view a copy of this license, visit http://creativecommons.org/licenses/by/4.0/.

(c) The Author(s) 2019 